\title{
Nanoparticles in Dentistry: Evidence and Future
}

\author{
Sydney B Gordon and Luciana M Shaddox* \\ Department of Periodontology, University of Kentucky College of Dentistry, USA
}

*Corresponding author: Luciana M Shaddox, Department of Periodontology, University of Kentucky College of Dentistry, 1095 VA dr. Lexington KY 40536, USA.

To Cite This Article: Sydney B Gordon, Luciana M Shaddox. Nanoparticles in Dentistry: Evidence and Future. 2020 - 8(4). AJBSR.MS.ID.001294. DOI: 10.34297/AJBSR.2020.08.001294.

Received: 阱 April 02, 2020; Published: 阱 April 16, 2020

\section{Introduction}

Currently, the medical field is using nanoparticle technology as a therapy treatment for drug delivery on a cellular level. Nanoparticles are between 1-100 nanometers (nm) and due to is size, penetrate deep into tissue and may delivery drugs crossing epithelial barriers [1]. They can possess anti-inflammatory characteristics and have been known to decrease inflammatory marker levels in animals in vivo [2]. Currently, the field of dentistry is using nanoparticles for implantation, drug delivery for caries treatment, and preventative care [3].

Mesoporous silica nanoparticles (MSN) are showing great potential in the process of drug delivery. The basic structure of MSN consists of a silica precursor, a surfactant, and a catalyst [4]. When synthesizing MSNs, manipulating variables such as temperature, $\mathrm{pH}$ level, concentrations of the silica, and surfactant will lead to the creation of different shapes and sizes. Nanoparticle templates can range from hard to soft, including vesicles, polymeric micelles, gold, or silica. Each type of template has a specific advantage over the other when considering the purpose of the nanoparticles. The mesopores can be loaded with different drugs, DNA, RNA, proteins, dyes, and metal nanoparticles.

Specific pore size, pH levels, and shape are essential for proper drug delivery systems. Each MSN can be manipulated to a particular size by adding suitable additive agents. The agents increase the kinetics of the reaction causing the MSN to become more compact. Depending on the $\mathrm{pH}$ needed for the specific drug delivery system, a base-catalyst or acid-catalyst will be utilized during the synthesis reaction. Cellular uptake of MSN is dependent on the functional properties of the nanoparticles [4]. Drug loading is determined by the characteristics of the nanoparticle, the drug, and the target release location. Overall, MSN has a high loading capacity, and HMSN (hollow mesoporous silica nanoparticles) has an even higher one due to the hollow cavities they contain. MSN have been loaded with anti-cancer, anti-inflammatory, antibacterial, antidepressant, osteogenic, antioxidant, and hypoglycemic drugs. These include ibuprofen (anti-inflammatory), erythromycin (antibiotic), doxorubicin (chemotherapy), and more [4].

Drug delivery is unique to each nanoparticle and is modified based on its cellular environment. The interaction between the surface pores and the drug molecules regulates the release of the cargo within the MSN. Specificity in drug delivery is accomplished through the distinct templates and drugs used in MSN synthesis. There are endless applications when it comes to drug delivery. MSN can be used for surface functionalization for enhanced drug loading, targeting antibodies, polymer coating, polyethylene glycol, and targeting ligands [4]. New gated release techniques are being researched to ensure that drug delivery does not occur before the MSN have reached their target destination. Some of these techniques include pH-responsive drug release, chemical and enzyme responsive drug release, and external stimuli for drug release [4].

\section{Uses in Dentistry}

The use of nanotechnology has multiple applications in Dentistry. In restorative dentistry, nanoparticles have been used to resist dental caries to prevent the failure of restorations [5]. These methods include antibacterial nanoparticles integrated into composite resin to aid in the reduction of biofilms. Silver and zinc-oxide nanoparticles were used and embedded into composite resin. Known caries causing bacterial species were evaluated around these composites as compared with non-nanoparticle containing resin. The use of zinc-oxide or silver nanoparticles in composite worked as an antibacterial agent to help prevent the growth of Streptococcus mutans and Lactobacillus [6]. Additionally, 
dimethylaminohexadecyl methacrylate (DMAHDM) nanoparticles were also added to restorative bonding agents to determine antibacterial properties against eight different periodontal biofilms. The results revealed reduced antibacterial growth while still providing adequate dentin bond strength [7].

Dental implants are being modified with antibacterial nanoparticles to reduce the probability of implant failure. Titanium implants infused with silver nanoparticles displayed significant antibacterial properties [8]. Nanoparticles are also being used in orthodontics, incorporated into orthodontic adhesive agents (cement), appliances, accessories (brackets, wires, etc.), and retainers [9-11], to prevent biofilm growth. Nanoparticles have also been combined into biomaterials for partial or complete dentures. Zinc-oxide nanoparticles (ZnOnps) were incorporated into polymethylmethacrylate (PMMA), the material used in dentures, and it was reported that PMMA with $2.5 \%$ ZnOnps had a significant antifungal effect [12]. Finally, nanoparticles have also been used as an antibacterial agent for oral prevention methods. For example, titanium dioxide nanoparticles were shown to be an active antimicrobial agent when incorporated into mouthwash [13]. Since it has proven antibacterial properties, MSN can also be altered for potential use as a local treatment for Periodontal disease. Periodontal disease is caused by a host reaction to a bacterial infection. The infection is often caused by a buildup of pathogenic biofilm in the gingival tissue. The disease has a variety of causes but is most commonly associated with dental plaque and other contributing factors that include smoking, diabetes mellitus, and drugs that can induce gingival hyperplasia [14]. This disease is prevalent, affecting 1 out of every 2 Americans [15]. If not treated, bone loss may result in tooth mobility and eventual tooth loss.

Current treatments of periodontal disease include scaling and root planning (mechanical debridement of the biofilm and calculus within the affected pockets), antibiotics/topical antimicrobials, and flap surgery in more severe cases. Scaling often does not remove all bacteria, which may result in a reoccurring infection. Current topical antimicrobial therapies have had limited effectiveness due to ineffective penetration into the tissue and easy wash out from the gingival pocket due to constant gingival crevicular fluid flow [16]. Systemic antibiotics provide some adjunctive benefit as well, however, an issue with antibiotic resistance is a concern [17]. Flap surgery may be conducted in more severe or recurring/refractory cases, to enable better access for debridement of the subgingival environment. This is usually an effective treatment, but it is costprohibitive to many patients.

As almost $50 \%$ of the population is affected by this disease, there is a substantial need for an efficient solution. Developing a localized treatment with nanoparticles could replace current costly therapies. Nanoparticles can be used for the delivery of antibiotics and anti-inflammatory agents directly within the subgingival environment. However, only minimal research has been conducted to test its effectiveness in eliminating bacteria and toxicity in the periodontium, none of which have researched the penetration through the epithelial cell barrier [18-20].

Nanoparticles can be used as a prevention method for multiple diseases of the mouth, including the treatment of oral cancer and gingivitis, bone replacement, stem cell imaging, and tracking, among others. The current implantation of this technology into the medical field shows tremendous potential for nanoparticles to become a part of everyday treatments. Since MSN have the potential to be customizable, the applications of nanoparticles in dentistry are endless.

\section{References}

1. Zhang X, Xu X, Li Y, Hu C, Zhang Z, et al. (2018) Virion-Like MembraneBreaking Nanoparticles with Tumor-Activated Cell-and-Tissue DualPenetration Conquer Impermeable Cancer. Adv Mater 30(27): e1707240.

2. Yilma AN, Singh SR, Dixit S, Dennis VA (2013) Anti-inflammatory effects of silver-polyvinyl pyrrolidone (Ag-PVP) nanoparticles in mouse macrophages infected with live Chlamydia trachomatis. Int J Nanomedicine 8: 2421-2432.

3. Abiodun Solanke I, Ajayi D, Arigbede A (2014) Nanotechnology and its application in dentistry. Ann Med Health Sci Res 4(Suppl 3): S171-S177.

4. Narayan R, Nayak UY, Raichur AM, Garg S (2018) Mesoporous Silica Nanoparticles: A Comprehensive Review on Synthesis and Recent Advances. Pharmaceutics 10(3): E118.

5. Hamilton MF, Otte AD, Gregory RL, Pinal R, Ferreira Zandona A, et al. (2015) Physicomechanical and antibacterial properties of experimental resin-based dental sealants modified with nylon-6 and chitosan nanofibers. J Biomed Mater Res B Appl Biomater 103(8): 1560-1568.

6. Shahin Kasraei, Lida Sami, Sareh Hendi, Mohammad Yousef AliKhani, Loghman Rezaei Soufi, et al. (2014) Antibacterial properties of composite resins incorporating silver and zinc oxide nanoparticles on Streptococcus mutans and Lactobacillus. Restor Dent Endod 39(2): 109114.

7. Wang L, Xie X, Weir MD, Fouad AF, Zhao L, et al. (2016) Effect of bioactive dental adhesive on periodontal and endodontic pathogens. J Mater Sci Mater Med 27(11): 168.

8. Li P, Tong Z, Huo L, Yang F, Su W (2016) Antibacterial and biological properties of biofunctionalized nanocomposites on titanium for implant application. J Biomater Appl 31(2): 205-214.

9. Metin Gursoy G, Taner L, Akca G (2017) Nanosilver coated orthodontic brackets: in vivo antibacterial properties and ion release. Eur J Orthod 39(1): 9-16.

10. Toodehzaeim MH, Zandi H, Meshkani H, Hosseinzadeh Firouzabadi A (2018) The Effect of CuO Nanoparticles on Antimicrobial Effects and Shear Bond Strength of Orthodontic Adhesives. J Dent (Shiraz) 19(1): 1-5.

11. Farhadian N, UsefiMashoof R, Khanizadeh S, Ghaderi E, Farhadian M, et al. (2016) Streptococcus mutans counts in patients wearing removable retainers with silver nanoparticles vs those wearing conventional retainers: A randomized clinical trial. Am J Orthod Dentofacial Orthop 149(2): 155-160.

12. Kamonkhantikul K, Arksornnukit M, Takahashi H (2017) Antifungal, optical, and mechanical properties of polymethylmethacrylate material incorporated with silanized zinc oxide nanoparticles. Int J Nanomedicine 12: 2353-2360. 
13. Ahrari F, Eslami N, Rajabi O, Ghazvini K, Barati S (2015) The antimicrobial sensitivity of Streptococcus mutans and Streptococcus sangius to colloidal solutions of different nanoparticles applied as mouthwashes. Dent Res J (Isfahan) 12(1): 44-49.

14. Bensley L, VanEenwyk J, Ossiander EM (2011) Associations of selfreported periodontal disease with metabolic syndrome and number of self-reported chronic conditions. Prev Chronic Dis 8(3): A50.

15. Eke PI, Dye BA, Wei L, Thornton-Evans GO, Genco RJ, Cdc Periodontal Disease Surveillance workgroup: James Beck, GDRP (2012) Prevalence of periodontitis in adults in the United States: 2009 and 2010. J Dent Res 91(10): 914-920.

16. Nair SC, Anoop KR (2012) Intraperiodontal pocket: An ideal route for local antimicrobial drug delivery. Journal of advanced pharmaceutical technology \& research 3(1): 9-15.
17. Heitz Mayfield LJ (2009) Systemic antibiotics in periodontal therapy. Aust Dent J 54(Suppl 1): S96-101.

18. LM Shaddox, H Huang, T Lin, W Hou, PL Harrison, I Aukhil, et al. (2012) Microbiological characterization in children with aggressive periodontitis. J Dent Res 91(10): 927-933.

19. Song W, Ge S (2019) Application of Antimicrobial Nanoparticles in Dentistry. Molecules 24(6): 1033.

20. Stefano G, Renna L, Brandizzi F (2012) Fluorescence-microscopy screening and next-generation sequencing: useful tools for the identification of genes involved in organelle integrity. J Vis Exp (62): 3809 . 